Tecno Lógicas

ISSN 0123-7799

Vol. 19, No. 36, pp. 13-25

Enero-junio de 2016



(C) Copyright 2015 por autores y Tecno Lógicas Este trabajo está licenciado bajo una Licencia Internacional Creative Commons Atribución (CC BY)

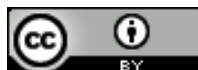

\section{Influencia de la forma de onda de pulsación en la estratificación de partículas de alta densidad en un equipo JIG}

\section{Influence of the pulse wave in the stratification of high density particles in a JIG device}

\author{
Manuel A. Ospina-Alarcón ${ }^{1}$, \\ Arturo B. Barientos-Ríos ${ }^{2}$ y Moisés O. Bustamante-Rua ${ }^{3}$
}

Recibido: 19 de febrero de 2015,

Aceptado: 31 de agosto de 2015

Cómo citar / How to cite

M.A. Ospina-Alarcón, A.B. Barientos-Ríos y M.O. Bustamante-Rua, "Influencia de la forma de onda de pulsación en la estratificación de partículas de alta densidad en un equipo JIG", Tecno Lógicas, vol. 19, no. 36 , pp. 13-25, 2016.

1 Ph.D. en Ingeniería - Ciencia y Tecnología de Materiales, Facultad de Ingenierías, Departamento de Mecatrónica y Electromecánica, Instituto Tecnológico Metropolitano, Medellín - Colombia, manuelospina@itm.edu.co

2 Ph.D. en Ciencias de la Ingeniería, Facultad de Ingeniería, Departamento de Ingeniería Metalúrgica, Universidad de Concepción, Concepción-Chile, abarrie@udec.cl

3 Ph.D. en Ingeniería Metalúrgica, Facultad de Minas, Departamento de Materiales y Minerales, Universidad Nacional de Colombia, Medellín-Colombia, mobustam@unal.edu.co 


\section{Resumen}

Se estudió el movimiento de partículas sometidas a cuatro perfiles de pulsación en un concentrador gravimétrico, mediante lechos fluidizados pulsados tipo jig, el cual es muy utilizado en la industria minera para la concentración de minerales pesados. Los perfiles de pulsación utilizados en la simulación fueron senoidal, triangular, diente de sierra atrasado y diente de sierra adelantado. Las velocidades locales bidimensionales del campo de flujo de agua se calcularon a partir de las ecuaciones de continuidad y momentum, por medio de técnicas CFD mediante la implementación del algoritmo SIMPLE. El movimiento de las partículas se modela mediante un balance de fuerzas aplicando la segunda ley de movimiento de Newton. Las fuerzas de interacción sólidolíquido se calculan mediante el modelo matemático Euleriano-Lagrangiano, extendido a una suspensión de partículas que poseen distribución amplia de tamaño y densidad. Para analizar el movimiento de las partículas, se deriva una ecuación de trayectoria que muestra la respuesta en el tiempo de las partículas, que incluye las fuerzas de masa virtual, gravedad, gradiente de presión, arrastre y Basset. El estudio mostró que la trayectoria de las partículas es muy sensible a la forma de onda aplicada en la condición de frontera a la entrada de la cámara del jig.

\section{Palabras clave}

Flujo sólido-líquido, concentración gravimétrica, modelo Euleriano - Lagrangiano, algoritmo SIMPLE, Jig.

\section{Abstract}

A study of particle motion subjected to four different pulsation profiles on a pulsated fluidized bed jig concentrator was carried out. The profiles used in the simulation were sinusoidal, triangle, sawtooth-backward and sawtooth-forward. Two-dimensional local velocities of the water flow field were calculated from the continuity and momentum equations by CFD techniques implementing SIMPLE algorithm. The particle motion is modeled by a forces balance applying the Newton's second law of motion. Liquid-solid interactions forces are calculated by the mathematical Euler-Lagrangian model extended to a particle suspension having a wide size and density distribution. To analyze the particle motion in jig, we derived a trajectory equation for the response time of particle that include virtual mass, gravity, pressure gradient, drag and Basset forces. The study demonstrates significant differences in the particle trajectories for various pulsation profiles applied to the boundary condition at the inlet to the jig chamber.

\section{Keywords} jig.

Solid-liquid flow, gravity concentration, Euler-Lagrange model, SIMPLE algorithm, 


\section{INTRODUCCIÓN}

El jig es uno de los equipos de concentración gravimétrica más antiguo usado en el procesamiento de minerales para separar oro, carbón, diamantes y otros minerales. Aunque en la actualidad los principios de funcionamiento básico no se han deducido completamente, es un equipo que se utiliza ampliamente en la separación de minerales a partir de la diferencia de tamaño o de densidad de las partículas [1]. El método consiste en aplicar un flujo de agua pulsante que dilata un lecho de partículas de densidad intermedia respecto a las partículas que se desean separar. Después de varios ciclos de pulsación, se obtiene la estratificación requerida de las partículas influenciadas por las fuerzas hidrodinámicas y la gravedad para cumplir finalmente con el requerimiento del producto deseado.



(a)

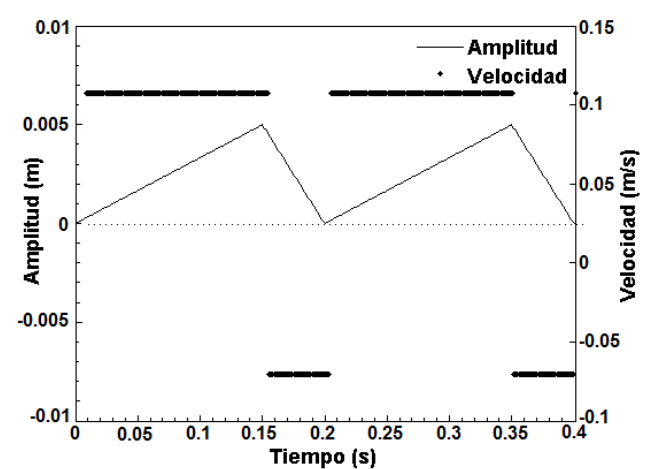

(c)
Existe en la literatura una gran cantidad de técnicas numéricas que se han utilizado para investigar el fenómeno de concentración en jigs. Estas técnicas de modelamiento asumen campo de flujo de líquido uniforme y no toman en cuenta el efecto de la velocidad no uniforme del agua sobre las fuerzas de interacción sólido-líquido. El modelo matemático EulerianoLagrangiano, propuesto inicialmente por Tsuji et al. [2], continúa siendo la técnica más llamativa debido a sus ventajas computacionales y a la capacidad que tiene de capturar la física de partículas. Esta técnica de modelamiento se está utilizando cada vez más en el estudio de equipos de concentración gravimétrica que utilizan flujos de agua pulsante [3]-[7]. Esta aproximación puede generar información detallada acerca de las trayectorias de las partículas y las fuerzas hidrodinámicas entre el agua y las partículas.

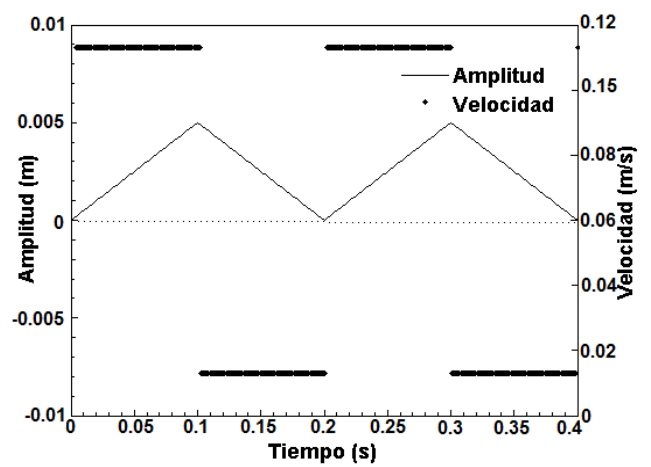

(b)

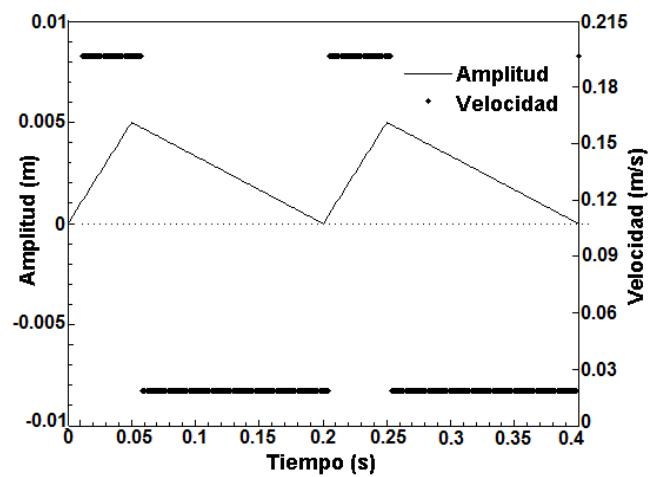

(d)

Fig. 1. Perfiles de pulsación aplicados en la condición de frontera a la entrada a) senoidal, b) triangular, c) diente de sierra adelantado, d) diente de sierra hacia atrás. Fuente: Autores 
Varios estudios han aplicado el modelo matemático Euleriano-Lagrangiano a jigs [3]-[6], [8]-[11]. Los estudios anteriores a excepción de Asakura et al. [8] han despreciado el efecto que tienen las fuerzas inerciales como la fuerza de masa virtual, gradiente de presión y Basset [12] sobre la trayectoria de las partículas, y solo Dong et al. [11] y Viduka et al. [4]-[6] han usado perfiles de pulsación diferente a uno senoidal. Ninguna investigación (incluyendo todas las diferentes técnicas de modelamiento) ha estudiado qué efecto tiene la forma del perfil de onda sobre los mecanismos de estratificación en partículas minerales que poseen una distribución amplia de tamaño, así como densidades relativas altas $(3<\mathrm{DR}<14)$, además de despreciar el efecto de las fuerzas inerciales en el modelo de trayectoria de partículas. Este artículo estudia cuatro perfiles de pulsación muy populares en este tipo de equipos (ver Fig. 1), con sistemas de partículas que poseen una distribución amplia de tamaño y densidad. El objetivo es determinar los diferentes comportamientos de las partículas alimentadas al jig sometidas a diferentes perfiles de pulsación, además de incluir las fuerzas inerciales al modelo de trayectoria.

\section{METOdOLOGíA}

El modelo del jig se basa en una descripción matemática EulerianaLagrangiana que toma en cuenta la interacción sólido-líquido. En este modelo, el balance de fuerzas sobre una partícula se extiende para calcular el efecto de la variación continua del agua sobre las partículas. El comportamiento del fluido es de carácter no estacionario y altamente turbulento. De esta manera el balance de fuerzas sobre una partícula alimentada al jig en forma vectorial es:

$$
m_{p_{i}} \frac{d u_{p i}}{d t}=f_{b}+f_{D}+f_{W M}+f_{p}+f_{B}+m_{p i} g
$$

En la ecuación (1) la fuerza total sobre una partícula se compone de una fuerza de cuerpo externa conocida (gravedad, mig), las fuerzas de interacción sólido-líquido (fb y fD) y las fuerzas inerciales (fVM, fP y fB). En la ecuación (1) mpi, upi, fb, fD, fVM, fP, $\mathrm{fB}$ son la masa de la partícula, la velocidad de la partícula, la fuerza de empuje, la fuerza de arrastre, la fuerza de masa virtual, la fuerza debida al gradiente de presión y la fuerza de Basset, respectivamente.

A continuación, se dará una breve descripción de la formulación del modelo que conduce al cálculo de las fuerzas que actúan sobre una partícula debido a su interacción con el fluido.

Siguiendo la correlación de Di Felice [13], la fuerza de arrastre fD es evaluada como:

$\mathbf{f}_{\mathrm{D}}=\frac{\pi}{8} \mathrm{C}_{\mathrm{D}} \rho_{\mathrm{l}} \mathrm{d}_{\mathrm{p}}^{2}\left|\mathbf{u}_{\mathrm{l}}-\mathbf{u}_{\mathrm{p}}\right|\left(\mathbf{u}_{\mathrm{l}}-\mathbf{u}_{\mathrm{p}}\right)$

donde $d_{p}$ es el diámetro de la partícula, $u_{p}$ es la velocidad de la partícula, $\rho$ l es la densidad del agua y $u_{1}$ es la velocidad del agua al interior de la cámara del jig. $\mathrm{C}_{\mathrm{D}}$ es el coeficiente de arrastre del agua, que para una partícula individual sin obstáculos se evalúa como:

$$
C_{D}=\frac{24}{R_{e p}}\left(1+0.15 R_{e p}^{0.697}\right)
$$

El número de Reynolds de la partícula, $R e_{p}$, se define en base a la velocidad relativa entre el agua y la partícula, como:

$R e_{p}=\frac{\rho 1\left|u_{1}-u_{p}\right| d_{p}}{\mu_{1}}$

donde $\mu_{1}$ es la viscosidad dinámica del agua.

Las fuerzas no estacionarias f $f_{\mathrm{MV}}$ y $\mathrm{f}_{\mathrm{p}} \mathrm{es}^{-}$ tán asociadas a la inercia y a la aceleración del fluido alrededor de las partículas. Ellas son llamadas la fuerza de masa virtual y la fuerza del gradiente de presión, respecti- 
vamente y se pueden calcular a partir del potencial de flujo que rodea las partículas [14].

$\mathbf{f}_{\mathrm{MV}}=\frac{1}{2} \rho_{\mathrm{l}} \mathrm{V}_{\mathrm{p}}\left(\frac{\mathrm{D} \mathbf{u}_{\mathrm{I}}}{\mathrm{Dt}}-\frac{\mathrm{d} \mathbf{u}_{\mathrm{p}}}{\mathrm{dt}}\right)$

$\mathbf{f}_{\mathbb{P P}}=-\rho_{1} V_{\mathrm{p}} \frac{\mathrm{D \mathbf {u } _ { \mathrm { I } }}}{\mathrm{Dt}}$

La expresión para la fuerza de Basset se obtiene mediante la siguiente integral de convolución [12]:

$$
\begin{aligned}
& f_{B}=-\frac{3}{2} \cdot d_{p}^{2}-\sqrt{\pi^{-} \cdot \mu_{1}^{-} P_{1}} \cdot \int_{0}^{t}\left(\frac{D u_{1}}{D t}-\frac{d u_{p}}{d t}\right) \frac{1}{\sqrt{t-\tau}} d \tau \\
& m_{p} \frac{d \mathbf{u}_{p}}{d t}=\left(\rho_{p}-\rho_{1}\right) v_{p} g \frac{\pi}{8} \rho_{1} d_{p}^{2} C_{d}\left(\mathbf{u}_{p}-\mathbf{u}_{1}\right)\left|\mathbf{u}_{p}-\mathbf{u}_{1}\right|-\frac{1}{2} \rho_{1} V_{p}\left(\frac{d \mathbf{u}_{p}}{d t}-\frac{D \mathbf{u}_{l}}{D t}\right)+\cdots \\
& \ldots-\rho_{1} V_{p} \frac{D u_{1}}{D t}-\frac{3}{2} \cdot d_{p}^{2} \cdot \sqrt{\pi \cdot \mu_{1} \cdot \rho_{1}} \cdot \int_{0}^{t}\left(\frac{D u_{1}}{D t}-\frac{d u_{p}}{d t}\right) \frac{1}{\sqrt{t-\tau}} d \tau
\end{aligned}
$$

El cálculo de la trayectoria de las partículas requiere la solución de dos ecuaciones diferenciales ordinarias, una para el cálculo de la velocidad y otra para el cálculo de la posición que en sus formas vectoriales son:

$\mathrm{m}_{\mathrm{p}_{\mathrm{i}}} \frac{\mathrm{d} \mathbf{u}_{\mathrm{p}}}{\mathrm{dt}}=\sum \mathbf{f}_{\mathrm{i}}$

$\frac{\mathrm{d} \mathbf{x}_{\mathrm{p}}}{\mathrm{dt}}=\mathbf{u}_{\mathrm{p}}$

donde $f_{i}$ representa las diferentes fuerzas que actúan sobre la partícula debida a la interacción viscosa con el agua. La trayectoria de las partículas se obtiene mediante integración numérica de (10) y (11).

El agua es tratada como una fase continua que se mueve a través de la cámara del jig y se modela de tal forma que la fracción volumétrica del agua modifica las ecuaciones estándar de Navier-Stokes. Así, las ecuaciones que gobiernan el movimiento del agua son la ecuación de continuidad donde $\tau$ es una variable de integración. Esta fuerza acopla la historia de la aceleración de la partícula con la viscosidad del fluido, indicando una difusión transiente de vórtice en el flujo [15]. Finalmente, la fuerza de empuje está dada por:

$\mathbf{f}_{\mathrm{b}}=\mathrm{V}_{\mathrm{p}} P_{1} \mathbf{g}$

donde $\mathrm{V}_{\mathrm{p}}$ es el volumen de la partícula y $\mathrm{g}$ es el vector de aceleración debida a la gravedad.

Las ecuaciones (2) a (8) se incorporan en (1) dando como resultado el modelo de trayectoria de las partículas y momentum, en términos de las variables medias locales sobre una celda computacional y son dadas en su forma vectorial por:

$$
\begin{aligned}
& \frac{\partial}{\partial t}\left(\rho_{1} \Phi_{1}\right)+\nabla \cdot\left(\rho_{1} \Phi_{1} \mathbf{u}_{1}\right)=0 \\
& \frac{\partial}{\partial t}\left(\rho_{1} \phi_{1} \mathbf{u}_{1}\right)+\frac{\partial}{\partial \mathrm{x}_{\mathrm{i}}}\left(\rho_{1} \phi_{1} \mathbf{u}_{1} \mathbf{u}_{1}\right)=-\nabla \mathrm{P}+\nabla\left(\phi_{1} \mathbf{\tau}\right)+\rho_{1} \phi_{1} \mathbf{g}+\frac{\sum_{\mathrm{i}=1}^{\mathrm{k}_{\mathrm{c}} \mathbf{f}_{\mathrm{wi}}}}{\Delta V}
\end{aligned}
$$
tensor de esfuerzos viscosos del fluido, la porosidad y el volumen de una celda computacional, respectivamente. El término de transferencia de momentum $f_{w i}$ en (13) relaciona la sumatoria de las fuerzas de interacción entre el agua y las partículas presentes en una celda computacional.

Las ecuaciones de movimiento de las partículas (10) y (11) se resuelve numéricamente con un código interno realizado en c/c++, que utiliza un método de integración explícito estableciendo condiciones geométricas y de frontera para el agua. El campo de velocidad para el agua (12) y (13) se 
resuelve usando un paquete de software comercial CFD (ANSYS Fluent 12.1). El acoplamiento entre la ecuación de movimiento de las partículas (9) y el campo de velocidad del agua se obtuvo de la siguiente manera: en cada intervalo de tiempo el marco Lagrangiano dará información de las posiciones y velocidades de las partículas individuales para la evaluación de la porosidad y las fuerzas hidrodinámicas volumétricas en una celda computacional, CFD usará posteriormente estos datos para determinar el campo de flujo del agua, que a su vez es utilizado para determinar las fuerzas hidrodinámicas que actúan sobre las partículas individuales. Incorporando las fuerzas resultantes en las ecuaciones de movimiento de las partículas (10) y (11) se obtiene información sobre la posición y la velocidad de las partículas individuales para el siguiente intervalo de tiempo. Las fuerzas hidrodinámicas que actúan sobre una partícula individual reaccionarán sobre el agua, cumpliéndose la tercera ley de movimiento de Newton.

Una vez se ha alcanzado convergencia de la solución numérica con cada uno de los diferentes perfiles de pulsación, las variables de salida de interés serán la posición tanto vertical como horizontal de las partículas y las fuerzas de interacción hidrodinámicas. Con esta información se analizará la trayectoria de las partículas, la posición vertical y la fuerza de interacción partícula fluido, variables significativas para entender la forma como las partículas se estratifican al interior de la columna del JIG.

\subsection{Condiciones de simulación}

El modelo del jig consiste de un dominio rectangular ocupado con partículas esféricas y líquido. Las partículas inyectadas poseen un tamaño entre $125 \mu \mathrm{m}$ a $2000 \mu \mathrm{m}$ y una densidad entre $3026 \mathrm{~kg} / \mathrm{m}^{3}$ a 13822 $\mathrm{kg} / \mathrm{m}^{3}$. En total se utilizaron 17 fracciones de densidad distribuidas en el intervalo
([3029-13822]). Una configuración detallada del modelo se muestra en la Tabla 1.

\begin{tabular}{lll}
\multicolumn{2}{c}{ Tabla 1. Especificaciones del modelo del jig. } \\
Fuente: Autores
\end{tabular}

Las paredes laterales se especificaron como condiciones de frontera no deslizante. La parte inferior de la columna rectangular se consideró condición de frontera de entrada de velocidad de agua y la parte superior como condición de frontera de salida de presión. La Fig. 2 muestra la columna del jig analizada.



Fig. 2. Geometría del dominio computacional. Fuente: Autores

El flujo de líquido se consideró bidimensional en el dominio computacional analizado, ya que, mediante un caso de simulación tridimensional previo, la variación del campo de velocidad mostró ser 
muy significativa en la dirección axial $\left(\mathrm{V}_{\mathrm{z}}\right)$ (ver Fig. 3), de esta manera se despreció el espesor de la columna del jig y solo se tomó en cuenta la altura y el ancho de la columna. Todos los perfiles de pulsación se estudiaron con un modelo bidimensional para lograr que los resultados sean aceptables para efectos de comparación.

El flujo de agua, el cual es variable con el tiempo, se inyecta a través de la entrada de la cámara del jig de acuerdo con el perfil de pulsación simulado. La entrada de flujo para el perfil de pulsación senoidal se estableció utilizando una ecuación de onda seno, mientras que los otros perfiles utilizan la función escalón (ver Fig. 1). Los perfiles de pulsación se comparan con base en que la amplitud y frecuencia de pulsación se mantienen constantes en $5 \mathrm{~mm}$ y $0.2 \mathrm{~s}$ (o 300 ciclos $/ \mathrm{min}$ ) respectivamente, y solamente se cambia la forma del perfil. Los perfiles usados y sus abreviaciones son: senoidal (SENO), triangular (TRI), diente de sierra adelantado (STF) y diente de sierra atrasado (STB). Cada perfil incluye un periodo de fluidización con un movimiento de líquido hacia arriba (valor positivo en la velocidad) y un periodo de succión con un movimiento de líquido hacia abajo (valor negativo de la velocidad). En todos los casos se utilizó la misma dimensión de la malla para asegurar resultados comparables independientes del tamaño de la malla.

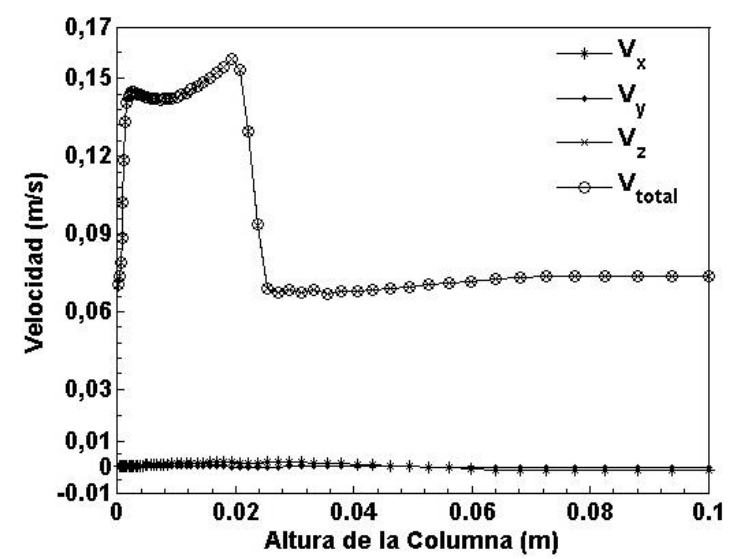

Fig. 3. Perfil de velocidad en las tres direcciones. Fuente: Autores

\section{RESULTADOS Y DISCUSIÓN}

\subsection{Trayectoria de partículas}

Inicialmente se revisan los patrones de trayectorias de partículas que pueden ayudar a entender el proceso de estratificación al interior de la cámara de separación del jig. Para todos los perfiles simulados, las partículas se inyectaron al interior de la columna del jig desde la misma posición $(\mathrm{x}=4.8 \mathrm{~cm}, \mathrm{y}=4.8 \mathrm{~cm})$ y a la misma veloci$\operatorname{dad}\left(\mathrm{V}_{\mathrm{x}}=5.56 \mathrm{~cm} / \mathrm{s}, \mathrm{V}_{\mathrm{y}}=0 \mathrm{~cm} / \mathrm{s}\right)$, además poseen un diámetro fijo de $300 \mu \mathrm{m}$ y una distribución de densidades en el intervalo de 3029 a 13822 kg/m³ . La Fig. 4 muestra la trayectoria que siguen las partículas en el interior de la columna del jig, las cuales se obtuvieron con todos los perfiles de pulsación para cuatro valores característicos de densidad. Todos los perfiles revelan diferencias significativas en cuanto a la estratificación (separación) de las partículas y al valor de densidad de corte a la cual las partículas se reportan en la zona de rechazo o concentrado.

En el ciclo de perfil senoidal (Fig. 4a) las partículas con una densidad mayor o igual a $3562 \mathrm{~kg} / \mathrm{m}^{3}$ se estratifican completamente; este comportamiento es muy diferente en todos los demás perfiles, donde solamente las partículas con una densidad mayor o igual a $4089 \mathrm{~kg} / \mathrm{m}^{3}$ se reportan en la corriente de concentrado (Fig. 4b a 4d). Un análisis general de la Fig. 4 muestra que el tiempo de residencia de las partículas en la cámara del jig es bastante sensible al perfil de pulsación aplicado, al tamaño y a la densidad de las partículas. Una partícula de densidad $\rho<3563 \mathrm{~kg} / \mathrm{m}^{3}$ saldrá más rápido cuando se le aplica un perfil STF que cuando se le aplica uno SENO. Caso contrario si la densidad es $\rho>3563 \mathrm{~kg} / \mathrm{m}^{3}$, la partícula permanecerá menor tiempo aplicándole un perfil SENO y se quedará un tiempo mayor con un perfil STF. 


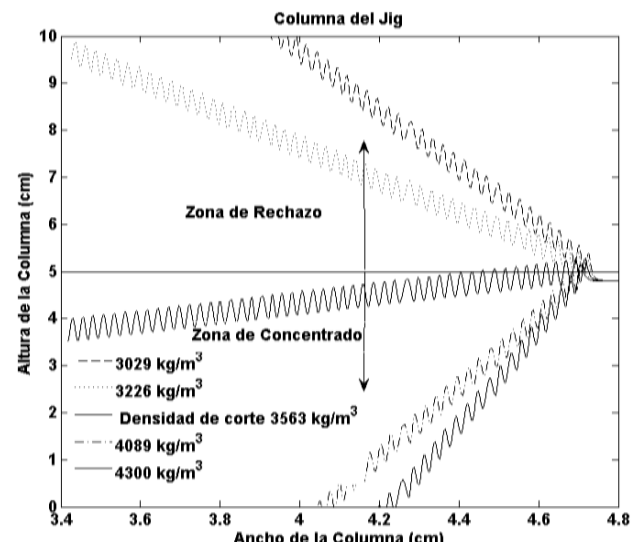

(a)

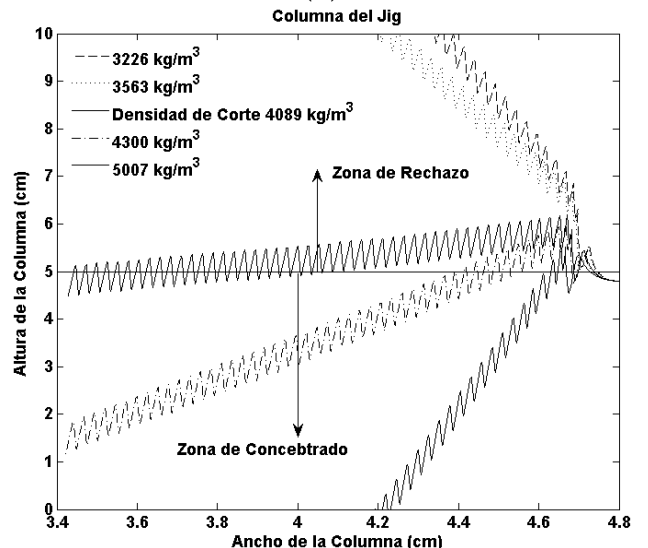

(c)

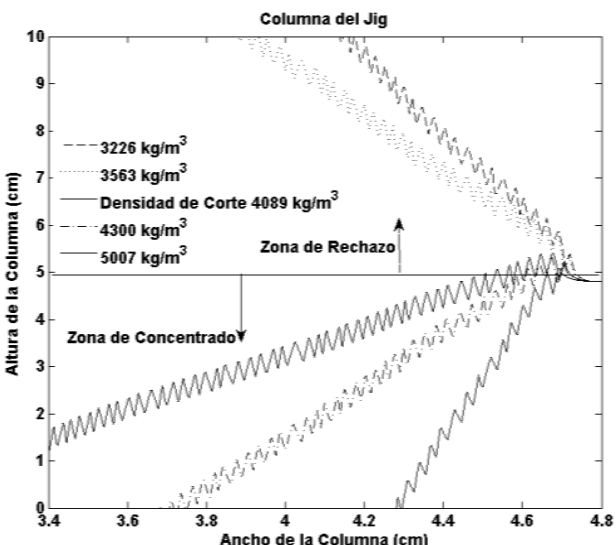

(b)

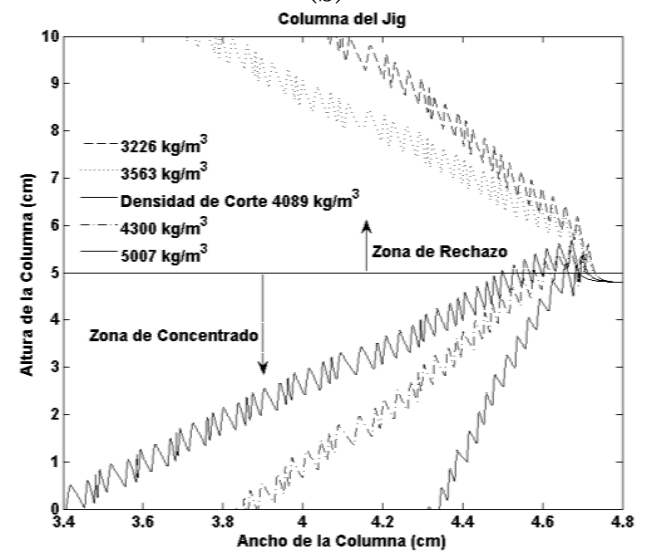

(d)

Figura 4. Patrones de trayectoria de partículas sometidos a los diferentes perfiles de pulsación. a) senoidal, b) triangular, c) diente de sierra adelantado, d) diente de sierra atrasado. Fuente: Autores

\subsection{Posición de las partículas}

La posición del desplazamiento vertical (ver Fig. 5) describe el comportamiento de las partículas al interior de la columna del jig, graficando para los diferentes tipos de partículas la altura que alcanzan en la columna del jig respecto al tiempo. Todos los perfiles muestran estratificación gradual. Las posiciones verticales revelan que las partículas alcanzan la altura de descarga (rechazo) $(6.5 \mathrm{~cm})$ de mayor a menor en el siguiente orden: diente de sierra atrasado (2.2 ciclos), diente de sierra adelantado (3 ciclos), triangular (3.5 ciclos) y senoidal (6 ciclos). Y la profundidad de concentrado $(3.5 \mathrm{~cm})$ de mayor a menor de la siguiente manera: senoidal (3.5 ciclos), triangular (5.5 ciclos), diente de sierra atrasado $(6.5$ ciclos) y diente de sierra ade- lantado (9.5 ciclos). Se demuestra que la amplitud de la posición de las partículas es directamente proporcional a la velocidad máxima de pulsación aplicada a la entrada. Es decir, las partículas tienen la misma frecuencia de pulsación (se conserva el periodo de oscilación de la forma de onda) pero su amplitud es proporcional al valor de la amplitud de la velocidad aplicada a la entrada de la cámara del jig con cada uno de los perfiles de pulsación utilizados (Ver Fig.1).

La posición en dirección vertical de cada tipo de partícula entrega una buena comprensión del proceso de concentración gravimétrica en jigs. Comenzando desde una condición inicial donde cada tipo de partícula es inyectada desde la misma posición y a la misma velocidad; las partículas livianas se mueven hacia arriba $\mathrm{mu}^{-}$ 
cho más rápido que las partículas pesadas durante el periodo de fluidización. Durante el periodo de succión, todas las partículas caen, pero las partículas más pesadas caen mucho más rápido que las livianas. Los resultados anteriores dan a entender que se pueden optimizar los perfiles de pulsación para mejorar el desempeño del equipo concentrador, en cuanto a la recuperación, razón de enriquecimiento y al consumo de tiempo y energía.

En la Fig. 5 se observa la diferencia de estratificación entre los perfiles de pulsación. Entre más grande sea la distancia de separación de las partículas livianas y pesadas, mayor será la estratificación en la columna del jig. Además, la Fig. 5 indica que todos los perfiles comienzan a estrati-

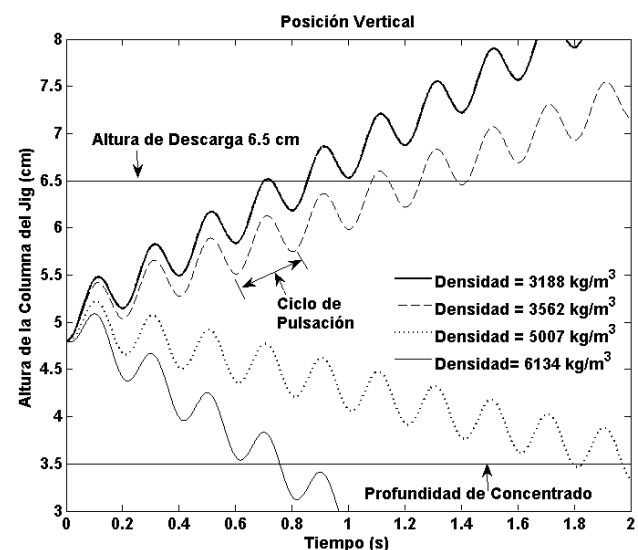

(a)

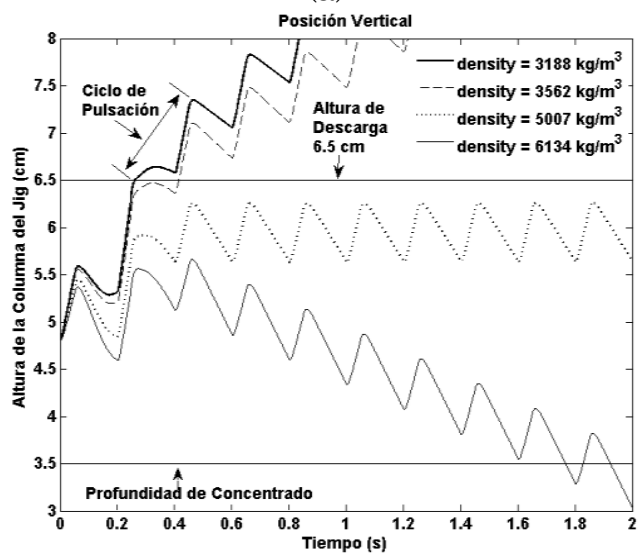

(c) ficar las partículas alrededor del segundo ciclo (0.4 segundos). Puede verse que después del cuarto ciclo de pulsación $\left(0.8\right.$ se- $^{-}$ gundos) las partículas están completamente estratificadas con todos los perfiles, pero con los perfiles triangular (Fig. 5b), diente de sierra adelantado (Fig. 5c) y diente de sierra atrasado (Fig. 5d) se necesitarán más ciclos de pulsación (más de 2 segundos) para lograr que las partículas de densidad $5007 \mathrm{~kg} / \mathrm{m}^{3}$ logren alcanzar la profundidad de concentrado. Este requerimiento de más ciclos de pulsación se ve reflejado en un mayor consumo de energía del dispositivo que genera la pulsación en el jig.



(b)

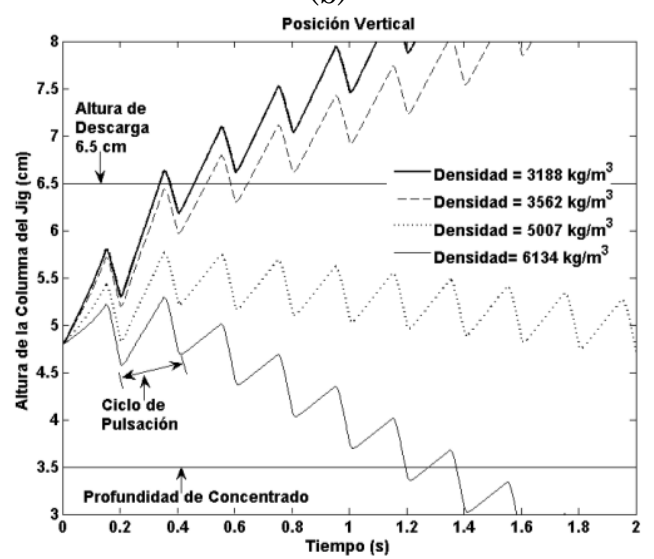

(d)

Fig. 5. Desplazamiento vertical de las partículas. a) senoidal, b) triangular, c) diente de sierra adelantado, d) diente de sierra atrasado. Fuente: Autores 


\subsection{Fuerzas de interacción partícula fluido}

La Fig. 6 entrega los valores de fuerza con perfil de pulsación senoidal, donde se indica si las partículas livianas o pesadas tienen suficiente fuerza de interacción partícula-fluido para superar su respectiva fuerza gravitacional y lograr un movimiento ascendente. La fuerza de interacción partícula-fluido adimensional $\left(\mathrm{f}_{\mathrm{p}-\mathrm{li}}\right)$ está dada como:

$\mathbf{f}_{\mathrm{p}-\mathrm{li}}=\left(\mathbf{f}_{\mathrm{b}, \mathrm{i}}+\mathbf{f}_{\mathrm{D}, \mathrm{i}}+\mathbf{f}_{\mathrm{VM}, \mathrm{i}}+\mathbf{f}_{\mathrm{VP}, \mathrm{i}}+\mathbf{f}_{\mathrm{B}, \mathrm{i}}\right) / \mathrm{m}_{\mathrm{i}} \mathbf{g} \quad(14)$

La ecuación (14) indica cual partícula (livianas o pesadas) tiene mayor aceleración.



(a)



(b)

Fig. 6. Fuerza de Interacción partícula-fluido adimensional (perfil senoidal). Fuente: Autores

Cuando la velocidad del agua asciende las partículas livianas tienen una fuerza ascendente más grande superando su fuerza gravitacional, mientras que las partículas pesadas tienen una fuerza ligeramente menor. A medida que avanzan los ciclos, este proceso se invierte gradualmente $\mathrm{de}^{-}$ bido a que las partículas livianas se mueven hacia la parte superior donde alcanzan la altura de rechazo, experimentando menor interacción hidrodinámica; al inicio del primer ciclo de pulsación las partículas livianas tienen una fuerza $\mathrm{f}_{\mathrm{p}-\mathrm{l}}>1$ resultando en una aceleración ascendente, mientras que las partículas pesadas tienen una fuerza $\mathrm{f}_{\mathrm{p}-\mathrm{l}}<1$, es decir, la fuerza de gravedad es mayor y las partículas pesadas co- $^{-}$ mienzan a descender con mayor velocidad de sedimentación depositándose en la parte inferior del lecho obteniéndose después del cuarto ciclo de pulsación una separación completa de las partículas.

El perfil triangular dado en la Fig. 7 muestra que los valores de $\mathrm{f}_{\mathrm{p}-1}$ de las partículas pesadas son más altos que el perfil senoidal, ya que la velocidad de entrada es más alta generando mayor interacción sólido-líquido. La diferencia entre los valores de $f_{p-1}$ de las partículas pesadas y livianas es muy pequeña para el perfil triangular, indicando que las partículas se desplazan casi de la misma forma al interior de la cámara del jig. Las partículas se estratifican rápidamente durante la etapa de fluidización, incrementando la interacción hidrodinámica y ocasionando mayor desplazamiento. Este comportamiento da lugar a un perfil de fuerza en forma de $\mathrm{S}$ (horizontalmente a lo largo del pico de fluidización).

Para un perfil diente de sierra atrasado, como se observa en la Fig. 8, en el periodo de succión durante la sedimentación, las partículas experimentan una fuerza de interacción positiva, empujando hacia arriba a las partículas livianas con más facilidad que las partículas pesadas, ya que las partículas livianas experimentan una fuerza positiva más alta cuando están cayendo. Esto ocurre a lo largo de cada ciclo, dando lugar a un ascenso pronunciado de las partículas, originando una estratificación debido a que las partículas pesadas tiene una gran oportunidad de pasar a través de las partículas livianas y sedimentarse. 




$3029 \mathrm{~kg} / \mathrm{m}^{3}-13823 \mathrm{~kg} / \mathrm{m}^{3}$

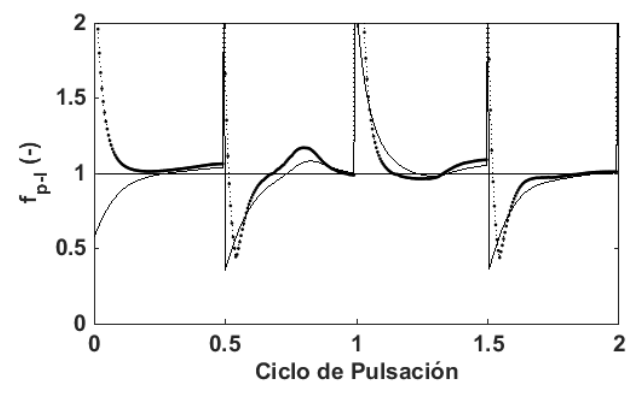

$3029 \mathrm{~kg} / \mathrm{m}^{3}-13823 \mathrm{~kg} / \mathrm{m}^{3}$

Fig. 7. Fuerza de Interacción partícula-fluido adimensional (Perfil triangular). Fuente: Autores
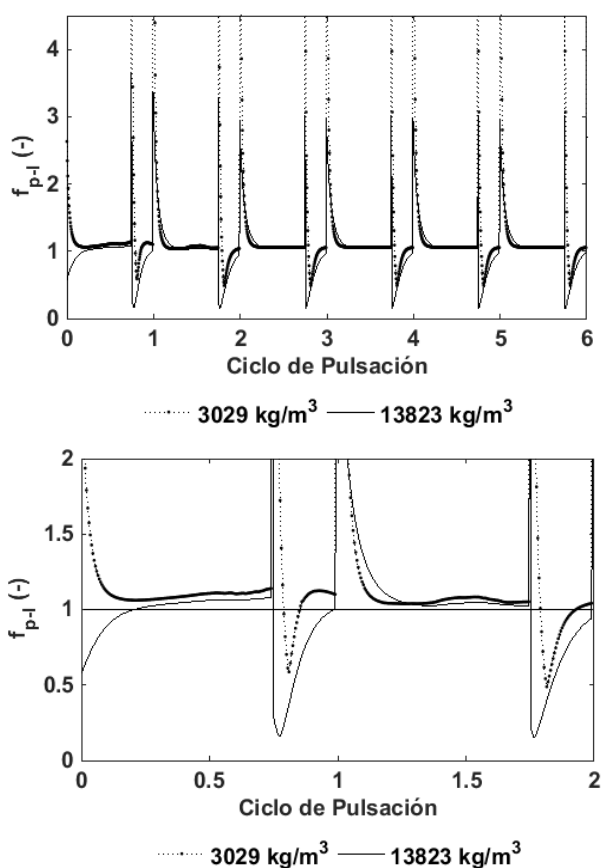

Fig. 8. Fuerza de Interacción partícula-fluido adimensional (Perfil diente de sierra atrasado). Fuente: Autores

Finalmente, los resultados de los valores de $f_{p-1}$ del perfil diente de sierra hacia adelante, mostrados en la Fig. 9, son típicos de un sistema de concentración gravi- métrica. Todos los valores de la interacción partícula-fluido oscilan alrededor de una media. Todas las partículas livianas tienen consistentemente un valor $\mathrm{f}_{\mathrm{p}-\mathrm{l}}>1$, esto guía a las partículas livianas hacia la corriente de rechazo. Las partículas pesadas están forzadas a moverse hacia abajo $\left(f_{p-1<1}\right)$ permitiendo el flujo ascendente de partículas livianas. Es fácil imaginar que el periodo de succión de este ciclo es redundante, ya que solamente existe una pequeña fracción de segundo para la caída de las partículas.

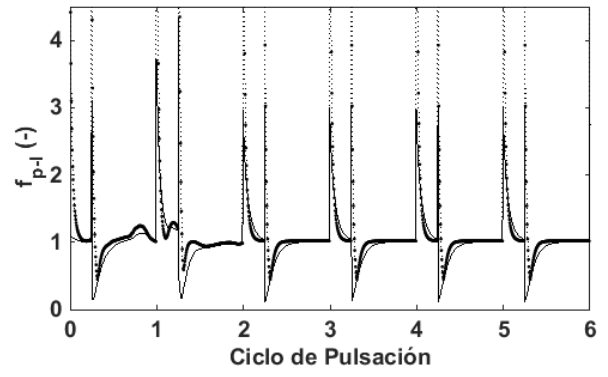

$3029 \mathrm{~kg} / \mathrm{m}^{3}-13823 \mathrm{~kg} / \mathrm{m}^{3}$

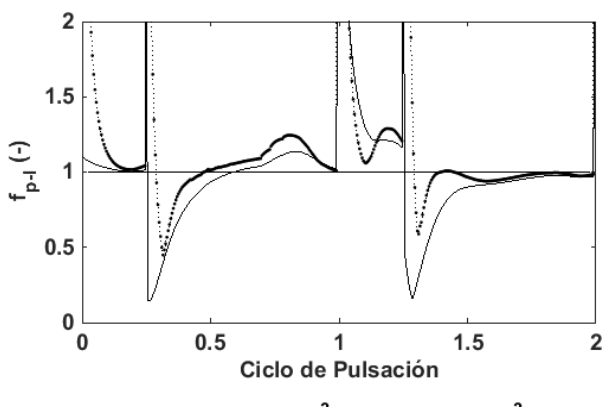

$3029 \mathrm{~kg} / \mathrm{m}^{3}-13823 \mathrm{~kg} / \mathrm{m}^{3}$

Fig. 9. Fuerza de Interacción partícula-fluido adimensional (perfil diente de sierra adelantado). Fuente: Autores

Cuando las partículas se están segregando y caen libremente sin obstaculizarse una contra otra, alcanzan eventualmente valores picos de $\mathrm{f}_{\mathrm{p}-\mathrm{l}} \sim 1$, esto muestra que las partículas caen o ascienden el tiempo suficiente logrando alcanzar su velocidad terminal, donde su peso es igual a las fuerzas hidrodinámicas (es decir, la aceleración es cero). Las partículas pesadas y livianas tienen diferentes valores de velocidad terminal que proporciona la concentración de las partículas alimentadas al jig. Los resultados muestran la necesidad de entender la interacción hidrodinámica entre las 
partículas y el fluido para lograr una buena optimización y desempeño del jig como equipo concentrador.

\section{CONCLUSIONES}

Se estudió la estratificación de partículas minerales en un jig bajo cuatro perfiles de pulsación usando el modelo matemático Euleriano-Lagrangiano. Con la amplitud y la frecuencia de pulsación en un valor constante, todos los cuatro perfiles de pulsación logran estratificar partículas minerales que poseen una distribución amplia de tamaño y densidad, pero sujetos a diferentes tiempos de separación (mayor tiempo de residencia para un perfil STF el cual se pude apreciar en la Fig. 4c).

La velocidad del agua a la entrada de la cámara del jig ocasionada por el perfil de pulsación afecta el movimiento de las partículas. Si en el inicio de la pulsación existe una velocidad del agua a la entrada muy alta, las partículas que alimentan al jig se mueven como un conjunto y muestran un comportamiento de transporte completo hacia la corriente de rechazo. Cuando la velocidad a la entrada se desarrolla lentamente, como en el perfil senoidal, las partículas no se levantarán como un conjunto y domina el levantamiento individual de las partículas más livianas al inicio del ciclo, permitiendo que las partículas pesadas se sedimenten y depositen en la corriente de concentrado. Además, si se adopta una velocidad de entrada lenta y constante ocurrirá la fluidización, como por ejemplo en el perfil diente de sierra hacia adelante.

La trayectoria de las partículas (Ver Fig. 4) y los desplazamientos verticales (Ver Fig. 5) mostraron que todos los perfiles de pulsación logran estratificar las partículas. Las partículas permanecen en una posición fija durante un intervalo de tiempo del periodo de succión en todos los perfiles de pulsación (debido a que las fuerzas hidrodinámicas se igualan con el peso de las partículas), demostrando las oportunidades de mejorar el proceso de concentración utilizando un perfil de pulsación optimizado, donde el periodo de succión del perfil pueda reducirse. Esta reducción del periodo de succión se reflejará en un menor consumo de energía por parte del equipo concentrador.

Los resultados indican que es necesario un estudio que involucre la interacción entre las partículas (fuerzas de interacción partícula -partícula o denominadas fuerzas de contacto sólido-sólido) para una comprensión más detallada del funcionamiento del equipo concentrador. Sin embargo, la investigación demuestra la utilidad del modelo Euleriano-Lagrangiano, como un modelo numérico efectivo para estudiar el proceso de concentración. Se requiere un trabajo adicional que considere diferentes amplitudes y periodos de pulsación para los cuatro perfiles, además de una validación con pruebas experimentales que ayude a entender y optimizar el desempeño del equipo mediante los principios físicos de conservación que lo rigen.

\section{REFERENCIAS}

[1] C. K. Gupta, Chemical Metallurgy Principles and Practice. Weinheim, 2003.

[2] Y. Tsuji, T. Kawaguchi, and T. Tanaka, "Discrete particle simulation of two-dimensional fluidized bed," Powder Technol., vol. 77, no. 1, pp. 79-87, Oct. 1993.

[3] F. W. Mayer, "Fundamentals of a potential theory of jigging process," in Proc. 7th Int. Miner. Proc. Cong., 1964, pp. 75-86.

[4] S. M. Viduka, Y. Q. Feng, K. Hapgood, and M. P. Schwarz, "CFDDEM Iinvestigation of particle separations using a trapezoidal jigging profile," in Ninth International Conference on CFD in the Minerals and Process Industries, CSIRO, 2012, no. December, pp. 1-8. 
[5] S. M. Viduka, Y. Q. Feng, K. Hapgood, and M. P. Schwarz, "CFDDEM investigation of particle separations using a sinusoidal jigging profile," $A d v$. Powder Technol., vol. 24, no. 2, pp. 473-481, Mar. 2013.

[6] S. M. Viduka, Y. Q. Feng, K. Hapgood, and M. P. Schwarz, "Discrete particle simulation of solid separation in a jigging device," Int. J. Miner. Process., vol. 123, pp. 108119, 2013.

[7] L. Dong, Y. Zhao, C. Duan, Z. Luo, B. Zhang, and X. Yang, "Characteristics of bubble and fine coal separation using active pulsing air dense medium fluidized bed," Powder Technol., vol. 257, pp. 40-46, May 2014.

[8] K. Asakura, M. Mizuno, M. Nagao, and S. Harada, "Numerical Simulation of Particle Motion in a Jig Separator," in 5th Join ASME JSME Fluids Engineering Conference, 2007, pp. 385-391.

[9] Y. K. Xia and F. F. Peng, "Numerical simulation of behavior of fine coal in oscillating flows," Miner. Eng., vol. 20, no. 2, pp. 113-123, Feb. 2007.
[10] Y. Xia, F. F. Peng, and E. Wolfe, "CFD simulation of fine coal segregation and stratification in jigs," Int. J. Miner. Process., vol. 82, no. 3, pp. 164-176, Apr. 2007.

[11] K. J. Dong, S. B. Kuang, a. Vince, T. Hughes, and a. B. Yu, "Numerical simulation of the in-line pressure jig unit in coal preparation," Miner. Eng., vol. 23, no. 4, pp. 301-312, 2010.

[12] A. B. Basset, A Treatise on Hidrodynamics Volumen II. London, 1888.

[13] R. Di Felice, "Hidrodynamics of liquid Fluidisation," Chem. Eng. Sci., vol. 50, no. 8, pp. 1213-1245, 1995.

[14] G. K. Batchelor, An Introduction to Fluid Dynamics. Cambridge, United Kingdom: Cambridge University Press, 1967.

[15] Y. D. Sobral, T. F. Oliveira, and F. R. Cunha, "On the unsteady forces during the motion of a sedimenting particle," Powder Technol., vol. 178, no. 2, pp. 129-141, 2007. 